\title{
Essai de psychoparodiologie trombono-assistée
}

\section{Eberhard Wolff}

PD Dr sc. soc., membre de la rédaction Histoire de la médecine

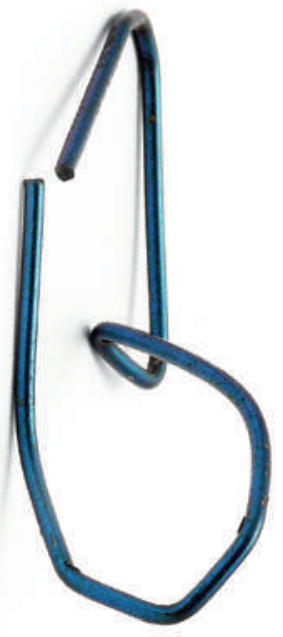

1 Gmür M. Büroklammern verbiegen. Zurich: Kein \& Aber; 2015.

2 www.gummibaerchen-orakel.ch, existe aussi sous forme de livre et d'app.

3 Seibt C. Dieser Artikel enthält Nacktfotos! Die Parodien der Nachrichten fangen an, die Nach richten zu ersetzen. Tages-Anzeiger du 10 avril 2015.

Crédit photographique (c) Zoltan Gabor / Kein \& Aber AG
«Le gris! ... mais pas trop gris quand même. Plutôt euh - gris-vert. Qui tire sur le brun. Une sorte de grisbrun avec du vert. Un mélange de gris-brun et de vert.» Peut-être vous rappelez-vous cette réponse de Monsieur Blömann à la psychologue qui lui demandait sa couleur préférée dans un sketch de Loriot datant des années 70 sur le conseil conjugal. On peut le voir aujourd'hui sur YouTube. «Une petite nuance de bleu ne gâcherait rien. Pourvu que ça reste gris. Gris-brun. On peut même ajouter une touche de rouge. Un rouge-brun. Mais dans l'ensemble, ça doit rester gris. Un gris vert-bleu et rougebrun, en somme.»

Excédée, la psychologue l'interrompt: «Pas besoin d'une telle précision, Monsieur Blömann!» Loriot rétorque sévèrement: «Oh, que si! Car après, vous irez vérifier dans vos tableaux ce que signifie le gris et vous lirez: Monsieur Blömann bat sa femme ou quelque chose comme ça. [...] Je connais ces pièges de la psychologie moderne. On dit une couleur et on se retrouve divorcé avec les torts.»

Près de quarante ans plus tard, le sketch de Loriot a repris vie dans un autre corps. Avec Büroklammern verbiegen («Plier des trombones»), le psychiatre et psychothérapeute zurichois Mario Gmür a publié un petit bestseller [1] où l'on découvre des photos fort esthétiques de trombones remodelés par les patientes et patients de son cabinet, poussés de toute évidence par une "pulsion primitive».

Ce qui n'était au départ qu'un projet artistique a été complété - sur volonté de l'éditeur - d'un test psychologique de personnalité qui constitue aujourd'hui la partie cardinale de l'ouvrage. Sans aller jusqu'à dire si nous battons ou non notre partenaire, cet autotest en plusieurs étapes prétend nous révéler, sur la base de nos manières favorites de plier les trombones, lesquels des 27 types de personnalité présentés correspondent à nos tendances ou du moins à nos aspirations: ambitieux, modeste, conservateur sceptique ou progressif confidentiel, pour ne citer que les quatre premiers. A en croire l'auteur, le livre nous en apprend autant que plusieurs semestres de psychologie. Une tentative héroïque de faire le test de personnalité dans notre famille a donné des résultats aussi concluants que «l'oracle des nounours en gélatine» pour celles et ceux qui connaissent ce jeu de société alémanique [2], ou, pour être plus explicite, du même niveau que les horoscopes des quotidiens gratuits. L'auteur trouve ses résultats "étonnamment proches de la vérité», tout en concédant qu'à ses yeux, le test n'est ni «d'un sérieux à toute épreuve», ni applicable dans la pratique médicale quotidienne. Son seul but est d'inciter chacun à étudier «ses propres traits et particularités psychiques».

Dès lors, l'ouvrage est-il sérieux ou ironique? La réponse est indéniablement: les deux! L'éditeur est connu pour des ouvrages qui naviguent gaillardement entre l'essai sérieux et la parodie complice, laissant le lecteur flotter dans un état intermédiaire entre sens et non-sens. C'est dans l'esprit du temps: l'art de la critique est aujourd'hui plus subtil qu'à l'époque de Loriot. L'ironie subversive est devenue l'instrument de communication du monde feutré de l'«infotainment». Constantin Seibt, du TagesAnzeiger, a dit récemment que la parodie surpassait désormais en crédibilité, en substance et en pertinence le compte-rendu sérieux de l'actualité [3].

Voici encore quelques décennies, un médecin qui aurait publié un tel ouvrage se serait probablement fait «briefer» par ses confrères de la société cantonale de médecine sur l'importance d'une image sérieuse du corps médical. Plus maintenant, j'en suis certain. L'esprit du temps aurait-il gagné la médecine, avec la psychologie en fer de lance? Dans ce cas, Peter Schneider, maître de la psychoironie, serait prophète dans sa propre Psychopolis.

Et si les résultats du test des trombones sont «étonnamment proches de la vérité», de quelle vérité parle-t-on? De celle des autres tests psychologiques? Quel crédit pouvons-nous encore donner à ces armes magiques de l'introspection humaine? Et à notre propre image? A l'ère du double langage, nos chances de le savoir diminuent de jour en jour. Nous ne serons plus jamais certains de lire un texte sérieux ou une parodie. Y compris dans cet article.

$\mathrm{Au}$ fait, cher Monsieur Gmür: d'après votre psycho-test $\mathrm{n}^{\circ} 1$, je suis à la fois honnête et prétentieux, frimeur et introverti. Est-ce que je risque la schizophrénie? D’après le test $\mathrm{n}^{\circ} 2$, je suis un «KLRT» pur beurre. Merci, cela m'avance beaucoup! Un «BCDFGNQUVW» m'aurait été tout aussi utile. Même Monsieur Blömann y aurait probablement trouvé son compte. Je suis sérieux! 